Research Article

\title{
Stochastic Logistic Systems with Jumps
}

\author{
Ruihua $W u^{1,2}$ and Ke Wang ${ }^{1}$ \\ ${ }^{1}$ Department of Mathematics, Harbin Institute of Technology (Weihai), Weihai 264209, China \\ ${ }^{2}$ College of Science, China University of Petroleum (East China), Qingdao 266555, China
}

Correspondence should be addressed to Ke Wang; wangke@hitwh.edu.cn

Received 18 July 2013; Accepted 25 December 2013; Published 12 January 2014

Academic Editor: Shiping Lu

Copyright (C) $2014 \mathrm{R}$. Wu and K. Wang. This is an open access article distributed under the Creative Commons Attribution License, which permits unrestricted use, distribution, and reproduction in any medium, provided the original work is properly cited.

\begin{abstract}
This paper is concerned with a stochastic nonautonomous logistic model with jumps. In the model, the martingale and jump noise are taken into account. This model is new and more feasible and applicable. Sufficient criteria for the existence of global positive solutions are obtained; then asymptotic boundedness in $p$ th moment, stochastically ultimate boundedness, and asymptotic pathwise behavior are to be considered.
\end{abstract}

\section{Introduction}

The well-known logistic system is an important and applicable system in both ecology and mathematical ecology. The classical nonautonomous logistic equation can be described by

$$
\mathrm{d} x(t)=x(t)[a(t)-b(t) x(t)] \mathrm{d} t
$$

for $t \geq 0$ with initial value $x(0)>0$. In this model, $x(t)$ is the population size at time $t$, so we are only concerned with positive solutions. The coefficient $a(t)$ is the intrinsic growth rate and $a(t) / b(t)$ stands for the carrying capacity at time $t$. Both $a(t)$ and $b(t)$ are continuous bounded functions on $t \geq 0$. System (1) models the population density of a single species whose members compete among themselves for a limited amount of food and living space. About the detailed model construction, readers can refer to [1].

Because of the importance in theory and practice, many authors have studied deterministic model (1) and its generalization. Many good results on the dynamical behavior have been reported; see, for example, Freedman and Wu [2], Lisena [3], Golpalsamy [4], Kuang [5], and the references therein. Among them, the books $[4,5]$ are good references in this area.

However, in the real world, the population systems are inevitably subject to much stochastic environmental noise which is important in ecosystem (see, e.g., Gard $[6,7]$ ).
In model (1), the parameters are all deterministic and irrespective of the environmental fluctuations; therefore, they have limitations in applications and it is difficult to fit data and predict the future accurately [8]. May [1] proposed the fact that because of the environmental noise, the birth rate, carrying capacity, and other parameters involved in the system exhibit random fluctuation to a greater or lesser extent. So it is necessary to find a more practical model.

According to the well-known central limit theorem, the sum of all stochastic environmental noise follows a normal distribution, and we usually call the white noise and denote it by $\dot{B}(t)$. We impose the stochastic perturbation on $b(t)$ and then get the following Itô's equation:

$$
\mathrm{d} x(t)=x(t)[(a(t)-b(t) x(t)) \mathrm{d} t+\sigma(t) x(t) \mathrm{d} B(t)],
$$

where $B(t)$ is a standard Brownian motion defined on a complete probability space $\left(\Omega, \mathscr{F},\left\{\mathscr{F}_{t}\right\}_{t \geq 0}, \mathbb{P}\right)$ with a filtration $\left\{\mathscr{F}_{t}\right\}_{t \geq 0}$ satisfying the usual conditions (i.e, $\mathscr{F}_{t}$ is right continuous and $\mathscr{F}_{0}$ contains all $\mathbb{P}$-null sets) and $\sigma^{2}(t)$ denotes the intensity of the noise. The white noise has important effects on the model. The famous result is that Mao et al. [9] showed that the environmental Brownian noise suppresses explosion in population dynamics. From then on, many valuable results are established. Liu and Wang studied the persistence and extinction of the solutions of model (2) in [10]. There are many other literatures to consider the models 
with white noise; the readers can refer to [11-22] and the references cited therein.

In addition, the population may suffer from sudden environmental shocks, namely, some jump type stochastic perturbation, for example, tsunami, earthquakes, floods, hurricanes, epidemics, red tides, and so on. These events are so strong that they break the continuity of the solution, so these phenomena cannot be described by (2). Introducing jump noise into the model may be a reasonable way to describe these phenomena; see $[23,24]$. Models with jumps have received considerable attention in recent years, but by now, there are few papers to deal with population systems driven by jumps. About the knowledge of jumps, the readers can refer to Applebaum [25] and Situ [26].

On the other hand, as is well known now, martingale is also an important field of stochastic analysis. Martingale theory has important and valuable applications in science, economics, finance, and elsewhere, and it has been developed well [26-28]. As we know that Brownian motion is a special martingale and it has many good properties, such as the independent increments, the increment stationary, and the Gaussian. So we attempt to generalize the driving process.

Motivated by the above reasons, it is eager to introduce a general driving process with jumps. In this paper, we propose and study the following logistic equation driven by martingales with jumps:

$$
\begin{gathered}
\mathrm{d} x(t)=x\left(t^{-}\right)\left[\left(a(t)-b(t) x\left(t^{-}\right)\right) \mathrm{d} t+\sigma(t) x\left(t^{-}\right) \mathrm{d} M(t)\right. \\
\left.+\int_{\mathbb{Z}} c\left(x\left(t^{-}\right), z\right) \widetilde{N}(\mathrm{~d} t, \mathrm{~d} z)\right] .
\end{gathered}
$$

In the model, $x\left(t^{-}\right)$is the left limit of $x(t), N$ is a Poisson counting measure with characteristic measure $\pi$ on a measurable subset $\mathbb{Z}$ of $(0, \infty)$ with $\pi(\mathbb{Z})<\infty$, and $\widetilde{N}(\mathrm{~d} t, \mathrm{~d} z)=$ $N(\mathrm{~d} t, \mathrm{~d} z)-\pi(\mathrm{d} z) \mathrm{d} t$ is the corresponding martingale measure. It is worth noting that a martingale usually does not share the good properties which a Brownian motion satisfies, so there are many difficulties when we extend the Brownian motion in the stochastic integral to the martingale; maybe this is one of the reasons why biological models driven by martingales have not been widely studied. About the detailed information of martingales, readers can refer to [26-28]. Throughout this paper, we assume that $M$ is independent of $N$.

The rest of this paper is organized as follows. In Section 2, sufficient conditions for global positive solutions are obtained. In Section 3, asymptotic boundedness in $p$ th moment and stochastically ultimate boundedness of model (3) are investigated. In Section 4, the asymptotic pathwise behavior is examined. We complete the paper with conclusions in Section 5.

\section{Global Positive Solutions}

Throughout this paper, we assume that $a(t), b(t)$, and $\sigma(t)$ are continuous bounded functions on $t \in \mathbb{R}_{+}=[0, \infty)$, $\inf _{t \in \mathbb{R}_{+}} b(t)>0 . M(t)$ denotes a square integrable martingale with $M(0)=0$, and $\langle M\rangle(t)$ is the unique integrable increasing process such that $M^{2}(t)-\langle M\rangle(t)$ is a martingale. Further, we assume that $\mathbb{E}\langle M\rangle(t)<\infty$, where $\mathbb{E}(f)$ means the mathematical expectation of $f$. In the sequel, we denote by $K$ a generic constant whose value may vary in different appearance.

Before we consider the asymptotic properties of solutions to system (3), first we should guarantee the existence of the global positive solutions. For the jump-diffusion coefficient $c(x, z)$, we impose the following conditions.

Assumption 1. For any $x \in \mathbb{R}_{+}$and $z \in \mathbb{Z}$,

$$
c(x, z)>-1,
$$

and, for each $k>0$, there exists a $L_{k}>0$ such that

$$
\int_{\mathbb{Z}}|c(x, z)-c(y, z)|^{2} \pi(\mathrm{d} z) \leq L_{k}|x-y|^{2},
$$

where $x, y \in \mathbb{R}_{+}$with $|x| \vee|y| \leq k$.

Theorem 2. Let Assumption 1 hold. And further assume that for $p \in(0,1)$ there exist constants $\delta>0, \alpha>1$ such that

$$
\int_{\mathbb{Z}}\left[(1+c(x, z))^{p}-1-p c(x, z)\right] \pi(d z) \leq-\delta|x|^{\alpha}+o\left(|x|^{\alpha}\right),
$$

for $x \in \mathbb{R}_{+}$, where $o\left(|x|^{\alpha}\right) /|x|^{\alpha} \rightarrow 0$ as $|x| \rightarrow \infty$. Then for any initial value $\bar{x}>0$, (3) has a unique global positive solution $x(t)$ for $t \geq 0$ almost surely.

Proof. Our proof is motivated by Bao and Yuan [24]. By (5), the coefficients of (3) satisfy the local Lipschitz condition, so for any initial value $\bar{x}>0$, (3) has a unique local solution $x(t)$ on $t \in\left[0, \tau_{e}\right)$, where $\tau_{e}$ is the exploded time (see [28]). To show that this solution is global, we only need to show that $\tau_{e}=\infty$ a.s. Let $k_{0}>0$ be sufficiently large satisfying $\bar{x}<k_{0}$. For each integer $k \geq k_{0}$, define an increasing sequence of stopping times $\tau_{k}$ by the prescription

$$
\tau_{k}=\inf \left\{t \in\left[0, \tau_{e}\right):|x(t)|>k\right\} .
$$

Clearly, $\tau_{k}$ is increasing as $k \rightarrow \infty$. Let $\tau_{\infty}=\lim _{k \rightarrow \infty} \tau_{k}$; then $\tau_{\infty} \leq \tau_{e}$. If we can obtain $\tau_{\infty}=\infty$ a.s., then $\tau_{e}=\infty$ a.s. In other words, if we can derive $\tau_{\infty}=\infty$, then we complete the proof. For $p \in(0,1)$, define a $C^{2}$-function $V: \mathbb{R}_{+} \rightarrow \mathbb{R}_{+}$ by

$$
V(x)=x^{p} .
$$


Let $T$ be an arbitrary positive constant, for $0 \leq t \leq \tau_{k} \wedge$ $T$, applying Itô's formula for semimartingales with jumps deduces that

$$
\begin{aligned}
\mathrm{d} V(x(t))= & p x^{p-1} x[(a(t)-b(t) x) \mathrm{d} t+\sigma(t) x \mathrm{~d} M(t)] \\
& +\frac{1}{2} p(p-1) x^{p-2} \sigma^{2}(t) x^{4} \mathrm{~d}\langle M\rangle(t) \\
& +\int_{\mathbb{Z}}\left[(x+x c(x, z))^{p}-x^{p}\right] \widetilde{N}(\mathrm{~d} t, \mathrm{~d} z) \\
& +\int_{\mathbb{Z}}\left[(x+x c(x, z))^{p}-x^{p}-p x^{p-1} x c(x, z)\right] \\
& \times \pi(\mathrm{d} z) \mathrm{d} t \\
= & p x^{p}[(a(t)-b(t) x) \mathrm{d} t+\sigma(t) x \mathrm{~d} M(t)] \\
& +\frac{1}{2} p(p-1) x^{p+2} \sigma^{2}(t) \mathrm{d}\langle M\rangle(t) \\
& +x^{p} \int_{\mathbb{Z}}\left[(1+c(x, z))^{p}-1\right] \widetilde{N}(\mathrm{~d} t, \mathrm{~d} z) \\
& +x^{p} \int_{\mathbb{Z}}\left[(1+c(x, z))^{p}-1-p c(x, z)\right] \pi(\mathrm{d} z) \mathrm{d} t \\
& +x^{p} \int_{\mathbb{Z}}\left[(1+c(x, z))^{p}-1\right] \widetilde{N}(\mathrm{~d} t, \mathrm{~d} z), \\
\leq & p x^{p}[a(t)-b(t) x] \mathrm{d} t+p x^{p+1} \sigma(t) \mathrm{d} M(t) \\
& +x^{p} \int_{\mathbb{Z}}\left[(1+c(x, z))^{p}-1\right] \widetilde{N}(\mathrm{~d} t, \mathrm{~d} z) \\
& +x_{\mathbb{Z}}^{p}\left[(1+c(x, z))^{p}-1-p c(x, z)\right] \pi(\mathrm{d} z) \mathrm{d} t \\
& L(t)) \mathrm{d} t+p x^{p+1} \sigma(t) \mathrm{d} M(t) \\
& \\
& \\
&
\end{aligned}
$$

where, for $x \in \mathbb{R}_{+}$,

$$
\begin{aligned}
L V(x)= & p x^{p}[a(t)-b(t) x] \\
& +x^{p} \int_{\mathbb{Z}}\left[(1+c(x, z))^{p}-1-p c(x, z)\right] \pi(\mathrm{d} z) \\
:= & K_{1}(x, p)+K_{2}(x, p) .
\end{aligned}
$$

Note that

$$
K_{1}(x, p) \leq K|x|^{p+1}+o\left(|x|^{p+1}\right) .
$$

By condition (6),

$$
K_{2}(x, p) \leq-\delta|x|^{p+\alpha}+o\left(|x|^{p+\alpha}\right) .
$$

So, for $\alpha>1$, there exists a $K>0$ such that

$$
L V(x) \leq K, \quad \forall x \in \mathbb{R}_{+} .
$$

For any given $l>0$, define

$$
\mu(l)=\inf \{V(x),|x| \geq l\} .
$$

Then we see that

$$
\lim _{l \rightarrow \infty} \mu(l)=\infty .
$$

By (9), (13), and the definition of $\mu$, we derive

$$
\begin{aligned}
\mu(k) \mathbb{P}\left(\tau_{k} \leq T\right) & \leq \mathbb{E}\left[V\left(x\left(\tau_{k}\right)\right) I_{\tau_{k} \leq T}\right] \leq \mathbb{E} V\left[x\left(\tau_{k} \wedge \mathrm{T}\right)\right] \\
& \leq K
\end{aligned}
$$

Letting $k \rightarrow \infty$ and making use of (15), we obtain

$$
\mathbb{P}\left(\tau_{\infty} \leq T\right)=0 .
$$

By the arbitrariness of $T$, we reach that

$$
\mathbb{P}\left(\tau_{\infty}=\infty\right)=1 \text {. }
$$

This completes the proof.

Remark 3. From the conditions and the results of Theorem 2, we can see that the jump processes can suppress the potential population explosion and guarantee the existence of the global solution.

Remark 4. The condition (6) can be achieved. Bao and Yuan [24] give an example to illustrate this.

\section{Asymptotic Moment Properties}

By now, we give the conditions to guarantee that (3) admits a unique global positive solution. From the biological point of view, the nonexplosion property and positivity in a population dynamical system are not good enough. In this section, we will examine the moment properties and stochastically ultimate boundedness.

Theorem 5. Let the conditions of Theorem 2 hold. Then for any $p \in(0,1)$, there exists a positive constant $K$ satisfying

$$
\limsup _{t \rightarrow \infty} \mathbb{E}|x(t)|^{p} \leq K
$$

Namely, (3) is asymptotically bounded in pth moment.

Proof. Let $V$ be defined as (8). For any $\bar{x}<k$, we define a stopping time sequence

$$
\sigma_{k}=\inf \{t \geq 0:|x(t)|>k\} .
$$

Clearly, $\sigma_{k}$ goes to infinity when $k \rightarrow \infty$. Applying Itô's formula leads to

$$
\begin{aligned}
& \mathbb{E}\left[e^{t \wedge \sigma_{k}} V\left(x\left(t \wedge \sigma_{k}\right)\right)\right] \\
& \quad \leq V(\bar{x})+\mathbb{E} \int_{0}^{t \wedge \sigma_{k}} e^{s}[V(x(s))+L V(x(s))] \mathrm{d} s,
\end{aligned}
$$

where $L V$ is defined by (10). Because the leading term of $L V(x)$ is $-\delta|x|^{\alpha+p}$ with $\alpha>1$ and $\delta>0$, then there exists a constant $K>0$ such that

$$
V(x)+L V(x) \leq K .
$$


Therefore,

$$
\mathbb{E}\left[e^{t \wedge \sigma_{k}} V\left(x\left(t \wedge \sigma_{k}\right)\right)\right] \leq V(\bar{x})+K\left(e^{t}-1\right) .
$$

Letting $k \rightarrow \infty$, we reach

$$
\mathbb{E}\left[e^{t} x^{p}(t)\right] \leq V(\bar{x})+K\left(e^{t}-1\right) .
$$

This is equivalent to

$$
\mathbb{E}\left[x^{p}(t)\right] \leq \frac{V(\bar{x})}{e^{t}}+K\left(1-e^{-t}\right) .
$$

Taking upper limit for both sides, we can get our desired results. This completes the proof.

As an application of Theorem 5, together with Chebyshev's inequality, we follow the next corollary.

Corollary 6. Under the conditions of Theorem 5, (3) is stochastically ultimate bounded. Namely, $\forall \epsilon \in(0,1), \exists H=$ $H_{\epsilon}>0$ such that

$$
\limsup _{t \rightarrow+\infty} \mathbb{P}[|x(t)|>H]<\epsilon,
$$

for any initial value $x(0)>0$.

\section{Asymptotic Pathwise Estimation}

In the previous section, we consider how the solutions vary on $\mathbb{R}_{+}$in probability or in moment. The pathwise properties of the solutions are the subject of the present section. For later applications, we first give a lemma which is a generalization of exponential martingale inequality with jumps [25].

Lemma 7. Let $f:[0, \infty) \rightarrow \mathbb{R}$ and $h:[0, \infty) \times \mathbb{Y} \rightarrow \mathbb{R}$ be both predictable $\left\{\mathscr{F}_{t}\right\}$-adapted processes such that for any $T>0$,

$$
\begin{gathered}
\int_{0}^{T}|f(t)|^{2} d\langle M\rangle(t)<\infty \text { a.s., } \\
\int_{0}^{T} \int_{\mathbb{Z}}|h(t, z)|^{2} \pi(d z) d t<\infty \text { a.s. }
\end{gathered}
$$

Then for any constants $\alpha, \beta>0$,

$$
\begin{aligned}
\mathbb{P}\left\{\sup _{0 \leq t \leq T}[\right. & \int_{0}^{t} f(s) d M(s)-\frac{\alpha}{2} \int_{0}^{t}|f(s)|^{2} d\langle M\rangle(s) \\
& +\int_{0}^{t} \int_{\mathbb{Z}} h(s, z) \widetilde{N}(d s, d z) \\
& \left.-\frac{1}{\alpha} \int_{0}^{t} \int_{\mathbb{Z}}\left[e^{\alpha h(s, z)}-1-\alpha h(s, z)\right] \pi(d z) d s\right] \\
>\beta & \leq e^{-\alpha \beta} .
\end{aligned}
$$

Proof. This part is motivated by [25]. For every integer $k \geq 1$, define the stopping time sequence

$$
\begin{aligned}
\rho_{k}=\inf \{ & t \geq 0:\left|\int_{0}^{t} f(s) \mathrm{d} M(s)\right|+\frac{\alpha}{2} \int_{0}^{t}|f(s)|^{2} \mathrm{~d}\langle M\rangle(s) \\
& +\left|\int_{0}^{t} \int_{\mathbb{Z}} h(s, z) \widetilde{N}(\mathrm{~d} s, \mathrm{~d} z)\right| \\
& \left.+\frac{1}{\alpha}\left|\int_{0}^{t} \int_{\mathbb{Z}}\left[e^{\alpha h(s, z)}-1-\alpha h(s, z)\right] \pi(\mathrm{d} z) \mathrm{d} s\right| \geq k\right\}
\end{aligned}
$$

and Itô's process

$$
\begin{aligned}
x_{k}(t)= & \alpha \int_{0}^{t} f(s) I_{\left[\left[0, \rho_{k}\right]\right]}(s) \mathrm{d} M(s) \\
& -\frac{\alpha^{2}}{2} \int_{0}^{t}|f(s)|^{2} I_{\left[\left[0, \rho_{k}\right]\right]}(s) \mathrm{d}\langle M\rangle(s) \\
& +\alpha \int_{0}^{t} \int_{\mathbb{Z}} h(s, z) I_{\left[\left[0, \rho_{k}\right]\right]}(s) \widetilde{N}(\mathrm{~d} s, \mathrm{~d} z) \\
& -\int_{0}^{t} \int_{\mathbb{Z}}\left[e^{\alpha h(s, z)}-1-\alpha h(s, z)\right] I_{\left[\left[0, \rho_{k}\right]\right]}(s) \pi(\mathrm{d} z) \mathrm{d} s .
\end{aligned}
$$

From the definition of $\rho_{k}$, we can see that $x_{k}(t)$ is bounded and $\rho_{k} \uparrow \infty$ a.s., when $k \rightarrow \infty$. Applying Itô's formula to $\exp \left[x_{k}(t)\right]$, we reach

$$
\begin{aligned}
e^{x_{k}(t)}= & +\alpha \int_{0}^{t} e^{x_{k}(s)} f(s) I_{\left[\left[0, \rho_{k}\right]\right]}(s) \mathrm{d} M(s) \\
& +\int_{0}^{t} \int_{\mathbb{Z}} e^{x_{k}(s)}\left(e^{\alpha h(s, z)}-1\right) I_{\left[\left[0, \rho_{k}\right]\right]}(s) \widetilde{N}(\mathrm{~d} s, \mathrm{~d} z) .
\end{aligned}
$$

This equality implies that $\exp \left[x_{k}(t)\right]$ is a local martingale with $\mathbb{E}\left(\exp \left[x_{k}(t)\right]\right)=1$, so $\exp \left[x_{k}(t)\right]$ is a martingale (see Theorem 5.2.4 in [25]). By virtue of Doob's martingale inequality, we derive

$$
\mathbb{P}\left(\sup _{0 \leq t \leq T} \exp \left[x_{k}(t)\right]>e^{\alpha \beta}\right) \leq e^{-\alpha \beta} \mathbb{E}\left(\exp \left[x_{k}(T)\right]\right)=e^{-\alpha \beta} .
$$

This is equivalent to

$$
\begin{aligned}
\mathbb{P}\left\{\sup _{0 \leq t \leq T}\right. & {\left[\int_{0}^{t} f(s) I_{\left[\left[0, \rho_{k}\right]\right]}(s) \mathrm{d} M(s)\right.} \\
& -\frac{\alpha}{2} \int_{0}^{t}|f(s)|^{2} I_{\left[\left[0, \rho_{k}\right]\right]}(s) \mathrm{d}\langle M\rangle(s)
\end{aligned}
$$




$$
\begin{gathered}
+\int_{0}^{t} \int_{\mathbb{Z}} h(s, z) I_{\left[\left[0, \rho_{k}\right]\right]}(s) \widetilde{N}(\mathrm{~d} s, \mathrm{~d} z) \\
-\frac{1}{\alpha} \int_{0}^{t} \int_{\mathbb{Z}}\left[e^{\alpha h(s, z)}-1-\alpha h(s, z)\right] I_{\left[\left[0, \rho_{k}\right]\right]}(s) \\
\times \pi(\mathrm{d} z) \mathrm{d} s]>\beta\} \leq e^{-\alpha \beta} .
\end{gathered}
$$

Let $k \rightarrow \infty$, we can arrive at our desired result. This completes the proof.

Theorem 8. Let the conditions of Theorem 2 hold. Assume further that there exist constants $\theta \in(0,1)$ and $\lambda>0$ such that

$$
\int_{\mathbb{Z}}\left[\ln ^{2}(1+c(x, z))+16 c(x, z)\right] \pi(d z) \leq \lambda|x|^{\theta}+o\left(|x|^{\theta}\right) .
$$

Then there exists a constant $K>0$, independent of initial value $\bar{x}>0$, such that the solution $x(t)$ of (3) satisfyies

$$
\limsup _{t \rightarrow \infty} \frac{\ln x(t)}{\ln t} \leq K \text { a.s. }
$$

Proof. By Theorem 2, (3) has a unique global positive solution for any initial condition $\bar{x}>0$. Applying Itô's formula to $\left[e^{t} \ln x(t)\right]$ leads to

$$
\begin{aligned}
e^{t} \ln x(t)-\ln \bar{x}= & \int_{0}^{t} e^{s}[\ln x(s)+a(s)-b(s) x(s)] \mathrm{d} s \\
& +\int_{0}^{t} e^{s} \sigma(s) x(s) \mathrm{d} M(s) \\
& -\frac{1}{2} \int_{0}^{t} e^{s} \sigma^{2}(s) x^{2}(s) \mathrm{d}\langle M\rangle(s) \\
& +\int_{0}^{t} \int_{\mathbb{Z}} e^{s} \ln (1+c(x, z)) \widetilde{N}(\mathrm{~d} s, \mathrm{~d} z) \\
& +\int_{0}^{t} \int_{\mathbb{Z}} e^{s}[\ln (1+c(x, z))-c(x, z)] \\
& \times \pi(\mathrm{d} z) \mathrm{d} s .
\end{aligned}
$$

By Lemma 7, for any $\alpha, \beta, T>0$, we have

$$
\begin{aligned}
\mathbb{P}\left\{\omega: \sup _{0 \leq t \leq T}\left[\int_{0}^{t} e^{s} \sigma(s) x(s) \mathrm{d} M(s)\right.\right. \\
-\frac{\alpha}{2} \int_{0}^{t} e^{2 s} \sigma^{2}(s) x^{2}(s) \mathrm{d}\langle M\rangle(s)
\end{aligned}
$$

$$
\begin{gathered}
+\int_{0}^{t} \int_{\mathbb{Z}} e^{s} \ln (1+c(x, z)) \widetilde{N}(\mathrm{~d} s, \mathrm{~d} z) \\
-\frac{1}{\alpha} \int_{0}^{t} \int_{\mathbb{Z}}\left[(1+c(x, z))^{\alpha e^{s}}\right. \\
\left.-1-\alpha e^{s} \ln (1+c(x, z))\right] \\
\times \pi(\mathrm{d} z) \mathrm{d} s]>\beta\} \leq e^{-\alpha \beta} .
\end{gathered}
$$

Take $T=k, \alpha=\varepsilon e^{-k}$, and $\beta=\left(2 e^{k} \ln k\right) / \varepsilon$, where $k \in \mathbb{N}$ and $0<\varepsilon<1 / 2$. By Borel-Cantalli's lemma, for almost all $\omega \in \Omega$, there is an integer $k_{0}=k_{0}(\omega)$ such that

$$
\begin{gathered}
\int_{0}^{t} e^{s} \sigma(s) x(s) \mathrm{d} M(s)+\int_{0}^{t} \int_{\mathbb{Z}} e^{s} \ln (1+c(x, z)) \widetilde{N}(\mathrm{~d} s, \mathrm{~d} z) \\
\leq \frac{2 e^{k} \ln k}{\varepsilon}+\frac{\varepsilon e^{-k}}{2} \int_{0}^{t} e^{2 s} \sigma^{2}(s) x^{2}(s) \mathrm{d}\langle M\rangle(s) \\
+\frac{1}{\varepsilon e^{-k}} \int_{0}^{t} \int_{\mathbb{Z}}\left[(1+c(x, z))^{\varepsilon e^{s-k}}-1\right. \\
\left.-\varepsilon e^{s-k} \ln (1+c(x, z))\right] \pi(\mathrm{d} z) \mathrm{d} s,
\end{gathered}
$$

for $0 \leq t \leq k$ and $k \geq k_{0}$. Therefore, for $0 \leq t \leq k$ and $k \geq k_{0}$,

$$
\begin{aligned}
\ln x(t) \leq & e^{-t} \ln \bar{x}+\int_{0}^{t} e^{s-t}[\ln x(s)+a(s)-b(s) x(s)] \mathrm{d} s \\
& +\frac{1}{2} \int_{0}^{t} e^{s-t} \sigma^{2}(s) x^{2}(s)\left[\varepsilon e^{s-k}-1\right] \mathrm{d}\langle M\rangle(s) \\
& +\frac{2 e^{k-t} \ln k}{\varepsilon}+\int_{0}^{t} \int_{\mathbb{Z}} e^{s-t}[\ln (1+c(x, z)) \\
& +\frac{e^{k-t}}{\varepsilon} \int_{0}^{t} \int_{\mathbb{Z}}\left[(1+c(x, z))^{\varepsilon e^{s-k}}-1\right. \\
\leq & e^{-t} \ln \bar{x}+\int_{0}^{t} e^{s-t}[\ln x(s)+a(s) \\
& \left.\quad-b(s) x(s)] \mathrm{d} s+\frac{2 e^{k-t} \ln k}{\varepsilon} \ln (1+c(x, z))\right] \pi(\mathrm{d} z) \mathrm{d} s \\
& +\frac{e^{k-t}}{\varepsilon} \int_{0}^{t} \int_{\mathbb{Z}}\left[(1+c(x, z))^{s e^{s-k}}-1\right. \\
:= & e^{-t} \ln \bar{x}+J_{1}(t)+\frac{2 e^{k-t} \ln k}{\varepsilon}+J_{2}(t),
\end{aligned}
$$


where in the second step we use the fundamental inequality $\ln x \leq x-1$, for $x>0$, and $\varepsilon e^{s-k}-1 \leq 0$. Making use of Taylor's series expansion, for $\varepsilon \in(0,1 / 2), x \in \mathbb{R}_{+}, z \in \mathbb{Z}$,

$$
\begin{aligned}
(1+c(x, z))^{\varepsilon e^{s-k}}= & 1+\varepsilon e^{s-k} \ln (1+c(x, z)) \\
& +\frac{\varepsilon^{2} e^{2(s-k)}}{2}(1+c(x, z))^{\xi} \ln ^{2}(1+c(x, z)),
\end{aligned}
$$

where $\xi \in(0, \varepsilon)$. Thus

$$
\begin{aligned}
J_{2}(t)=\int_{0}^{t} \int_{\mathbb{Z}} & \frac{\varepsilon e^{2 s-k-t}}{2}(1+c(x, z))^{\xi} \ln ^{2}(1+c(x, z)) \\
& \times \pi(\mathrm{d} z) \mathrm{d} s .
\end{aligned}
$$

For $t \leq k$ and $k \geq k_{0}$, we have

$$
\begin{gathered}
J_{2}(t)=\int_{0}^{t} \int_{-1<c(x, z)<0} \frac{\varepsilon e^{2 s-k-t}}{2}(1+c(x, z))^{\xi} \\
\quad \times \ln ^{2}(1+c(x, z)) \pi(\mathrm{d} z) \mathrm{d} s \\
+\int_{0}^{t} \int_{c(x, z) \geq 0} \frac{\varepsilon e^{2 s-k-t}}{2}(1+c(x, z))^{\xi} \\
\quad \times \ln ^{2}(1+c(x, z)) \pi(\mathrm{d} z) \mathrm{d} s \\
:=J_{21}(t)+J_{22}(t) .
\end{gathered}
$$

For $-1<c(x, z)<0$ and $0<\xi<\varepsilon<1 / 2$, we have $(1+c)^{\xi}<1$. So

$$
\begin{aligned}
J_{21}(t)<\int_{0}^{t} \int_{-1<c(x, z)<0} \frac{\varepsilon e^{2 s-k-t}}{2} \\
\quad \times \ln ^{2}(1+c(x, z)) \pi(\mathrm{d} z) \mathrm{d} s \\
\leq \int_{0}^{t} \int_{\mathbb{Z}} \frac{\varepsilon e^{2 s-k-t}}{2} \ln ^{2}(1+c(x, z)) \pi(\mathrm{d} z) \mathrm{d} s .
\end{aligned}
$$

By virtue of the fundamental inequality $(\ln x \leq x-1, x>0)$

$$
\frac{1}{4} \ln x=\ln x^{1 / 4} \leq x^{1 / 4}-1
$$

and noting that $(1+c)^{\xi} \leq(1+c)^{1 / 2}$ when $c(x, z) \geq 0$ and $0 \leq \xi<\varepsilon<1 / 2$, we see that

$$
\begin{aligned}
J_{22}(t) & \leq 16 \int_{0}^{t} \int_{c(x, z) \geq 0} \frac{\varepsilon e^{2 s-k-t}}{2}(1+c(x, z)) \pi(\mathrm{d} z) \mathrm{d} s \\
& \leq 16 \int_{0}^{t} \int_{\mathbb{Z}} \frac{\varepsilon e^{2 s-k-t}}{2}(1+c(x, z)) \pi(\mathrm{d} z) \mathrm{d} s .
\end{aligned}
$$

Substituting (44) and (46) into (43), for $t \leq k$ and $k \geq k_{0}$, we find

$$
\begin{aligned}
J_{2}(t) \leq \frac{\varepsilon}{2} \int_{0}^{t} \int_{\mathbb{Z}} e^{s-t} & {\left[\ln ^{2}(1+c(x, z))\right.} \\
& +16(1+c(x, z))] \pi(\mathrm{d} z) \mathrm{d} s
\end{aligned}
$$

By condition (34), there exists a constant $K>0$ such that

$$
J_{1}(t)+J_{2}(t) \leq K\left(1-e^{-t}\right)
$$

Substituting (48) into (39), for any $\omega \in \Omega, 0 \leq t \leq k$, and $k \geq k_{0}$, we arrive at

$$
\ln x(t) \leq e^{-t} \ln \bar{x}+\frac{2 e^{k-t} \ln k}{\varepsilon}+K .
$$

Therefore, for $k-1 \leq t \leq k$, we have

$$
\frac{\ln x(t)}{\ln t} \leq \frac{\ln \bar{x}}{e^{t} \ln t}+\frac{K}{\ln t}+\frac{2 e \ln k}{\varepsilon \ln (k-1)} .
$$

Letting $k \rightarrow \infty$ (then $t \rightarrow \infty$ ), we follow that

$$
\limsup _{t \rightarrow+\infty} \frac{\ln x(t)}{\ln t} \leq \frac{2 e}{\varepsilon} \text {. }
$$

Letting $\varepsilon \uparrow 1 / 2$, we can obtain our desired assertion. This completes the proof.

Remark 9. The condition (34) can be fulfilled; Bao and Yuan [24] give an example to show this.

Using the equality $\lim _{t \rightarrow \infty}(\ln t) / t=0$ and Theorem 8 , we follow that the sample Lyapunov exponent of (3) is less than or equal to zero, which is described by the following corollary.

Corollary 10. Under the conditions of Theorem 8, one has

$$
\limsup _{t \rightarrow+\infty} \frac{\ln x(t)}{t} \leq 0 \text { a.s. }
$$

\section{Conclusions}

In this paper, we consider the stochastic logistic system driven by martingales with jumps. The martingale and jumps make our study more difficult. For the system, sufficient criteria for the existence of a global positive solution are obtained; then asymptotic boundedness, in pth moment, stochastically ultimate boundedness and asymptotic pathwise behavior are considered. The results strictly generalize the existing results, so it is meaningful and important.

\section{Conflict of Interests}

The authors declares that there is no conflict of interests regarding the publication of this paper.

\section{Acknowledgments}

The authors thank the editor and referees for their very important and helpful comments and suggestions. They also thank the National Natural Science Foundation of PR China (Nos. 11171081 and 11171056).

\section{References}

[1] R. M. May, Stability and Complexity in Model Ecosystems, Princeton University Press, 1973. 
[2] H. I. Freedman and J. Wu, "Periodic solutions of single-species models with periodic delay," SIAM Journal on Mathematical Analysis, vol. 23, no. 3, pp. 689-701, 1992.

[3] B. Lisena, "Global attractivity in nonautonomous logistic equations with delay," Nonlinear Analysis: Real World Applications, vol. 9, no. 1, pp. 53-63, 2008.

[4] K. Golpalsamy, Stability and Oscillations in Delay Differential Equations of Population Dynamics, Kluwer Academic, Dordrecht, The Netherlands, 1992.

[5] Y. Kuang, Delay Differential Equations With Applications in Population Dynamics, Academic Press, Boston, Mass, USA, 1993.

[6] T. C. Gard, "Persistence in stochastic food web models," Bulletin of Mathematical Biology, vol. 46, no. 3, pp. 357-370, 1984.

[7] T. C. Gard, "Stability for multispecies population models in random environments," Nonlinear Analysis, vol. 10, no. 12, pp. 1411-1419, 1986.

[8] M. Bandyopadhyay and J. Chattopadhyay, "Ratio-dependent predator-prey model: effect of environmental fluctuation and stability," Nonlinearity, vol. 18, no. 2, pp. 913-936, 2005.

[9] X. Mao, G. Marion, and E. Renshaw, "Environmental Brownian noise suppresses explosions in population dynamics," Stochastic Processes and their Applications, vol. 97, no. 1, pp. 95-110, 2002.

[10] M. Liu and K. Wang, "Persistence and extinction in stochastic non-autonomous logistic systems," Journal of Mathematical Analysis and Applications, vol. 375, no. 2, pp. 443-457, 2011.

[11] D. Jiang and N. Shi, "A note on nonautonomous logistic equation with random perturbation," Journal of Mathematical Analysis and Applications, vol. 303, no. 1, pp. 164-172, 2005.

[12] D. Jiang, N. Shi, and X. Li, "Global stability and stochastic permanence of a non-autonomous logistic equation with random perturbation," Journal of Mathematical Analysis and Applications, vol. 340, no. 1, pp. 588-597, 2008.

[13] X. Li, A. Gray, D. Jiang, and X. Mao, "Sufficient and necessary conditions of stochastic permanence and extinction for stochastic logistic populations under regime switching," Journal of Mathematical Analysis and Applications, vol. 376, no. 1, pp. 1128, 2011.

[14] M. Liu and K. Wang, "Asymptotic properties and simulations of a stochastic logistic model under regime switching," Mathematical and Computer Modelling, vol. 54, no. 9-10, pp. 2139-2154, 2011.

[15] X. Li, D. Jiang, and X. Mao, "Population dynamical behavior of Lotka-Volterra system under regime switching," Journal of Computational and Applied Mathematics, vol. 232, no. 2, pp. 427-448, 2009.

[16] M. Liu and K. Wang, "On a stochastic logistic equation with impulsive perturbations," Computers and Mathematics with Applications, vol. 63, no. 5, pp. 871-886, 2012.

[17] M. Liu and K. Wang, "Asymptotic properties and simulations of a stochastic logistic model under regime switching II," Mathematical and Computer Modelling, vol. 55, no. 3-4, pp. 405$418,2012$.

[18] M. Liu and K. Wang, "Population dynamical behavior of LotkaVolterra cooperative systems with random perturbations, DiscreteContin," Journal of Dynamic Systems, vol. 33, pp. 24952522, 2013.

[19] W. Li, H. Su, and K. Wang, "Global stability analysis for stochastic coupled systems on networks," Automatica, vol. 47, no. 1, pp. 215-220, 2011.
[20] W. Li, H. Song, Y. Qu, and K. Wang, "Global exponential stability for stochastic coupled systems on networks with Markovianswitching," Systems \& Control Letters, vol. 62, no. 6, pp. 468-474, 2013.

[21] M. Liu and K. Wang, "Analysis of a stochastic autonomous mutualism model," Journal of Mathematical Analysis and Applications, vol. 402, no. 1, pp. 392-403, 2013.

[22] M. Liu and K. Wang, "Stochastic Lotka-Volterra systems with Lévy noise," Journal of Mathematical Analysis and Applications, vol. 410, no. 2, pp. 750-763, 2014.

[23] J. Bao, X. Mao, G. Yin, and C. Yuan, "Competitive LotkaVolterra population dynamics with jumps," Nonlinear Analysis, Theory, Methods and Applications, vol. 74, no. 17, pp. 6601-6616, 2011.

[24] J. Bao and C. Yuan, "Stochastic population dynamics driven by Lévy noise," Journal of Mathematical Analysis and Applications, vol. 391, no. 2, pp. 363-375, 2012.

[25] D. Applebaum, Lévy Process and Stochastic Calculus, Cambridge University Press, 2nd edition, 2009.

[26] R. Situ, Theory of Stochastic Differential Equation With Jumps and Applications, Springer, New York, NY, USA, 2012.

[27] Z. Huang, The Introduction To STochastic Analysis, Beijing, China, 2001, chinese.

[28] G. Gong, The Introduction to Stochastic Differential Equations, Beijing, China, 1995, chinese. 


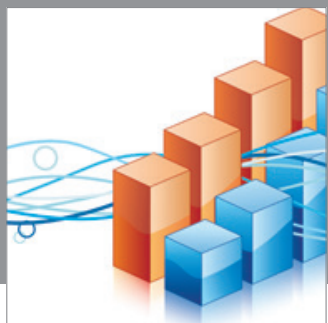

Advances in

Operations Research

mansans

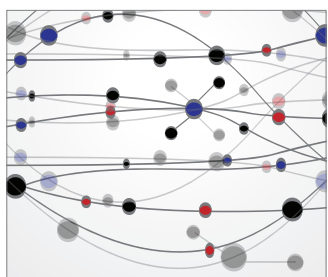

The Scientific World Journal
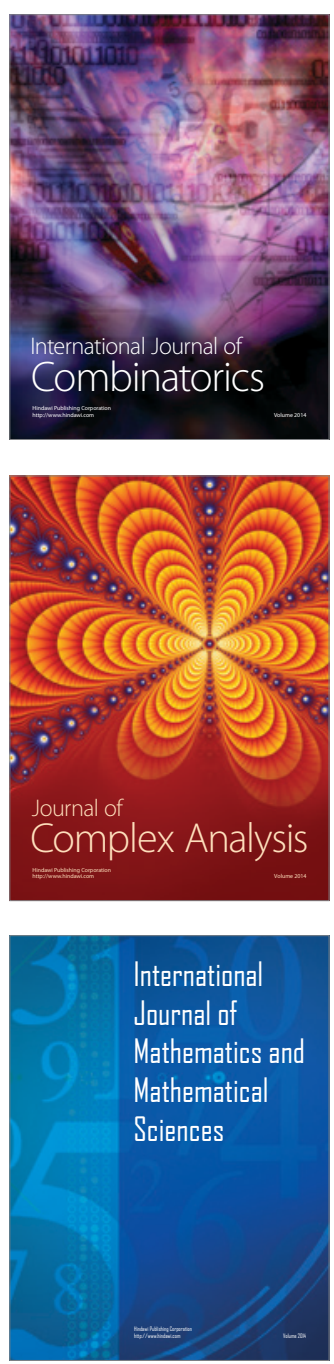
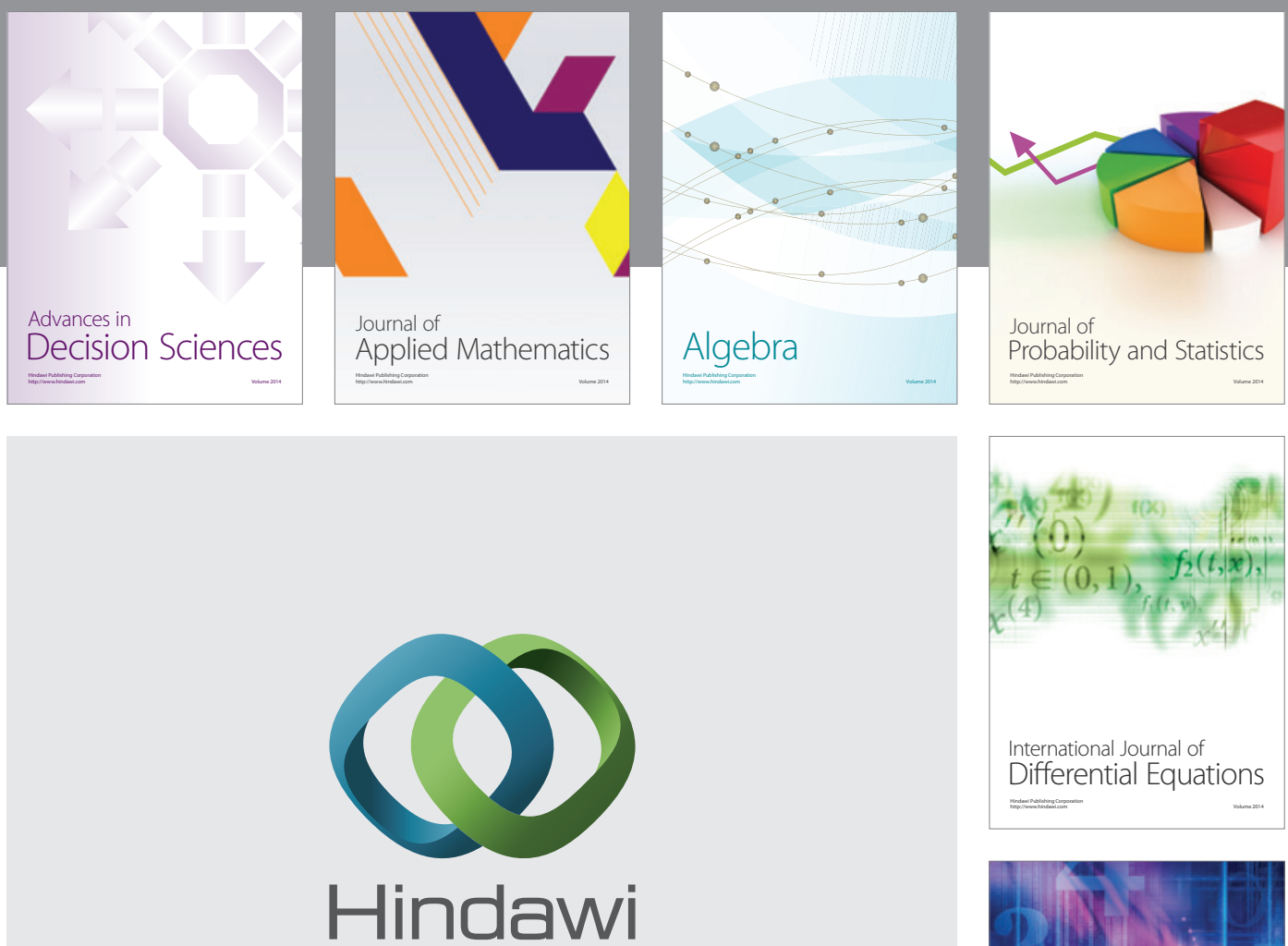

Submit your manuscripts at http://www.hindawi.com
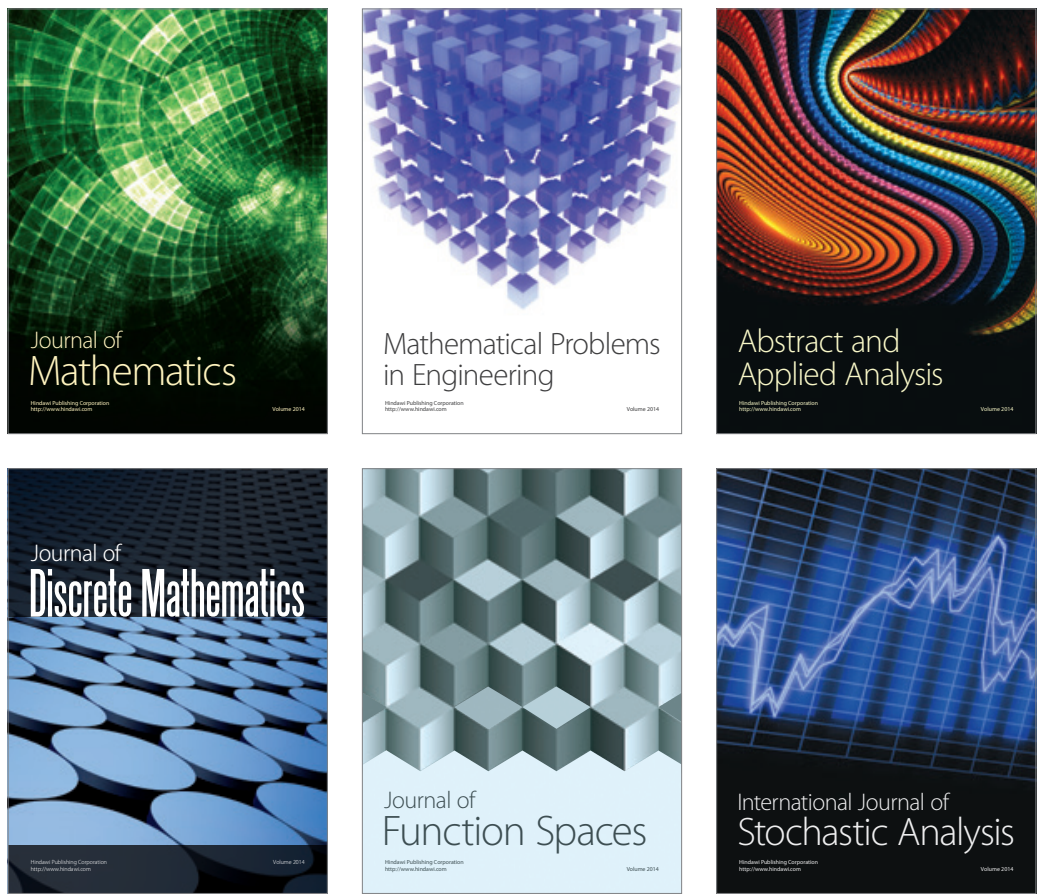

Journal of

Function Spaces

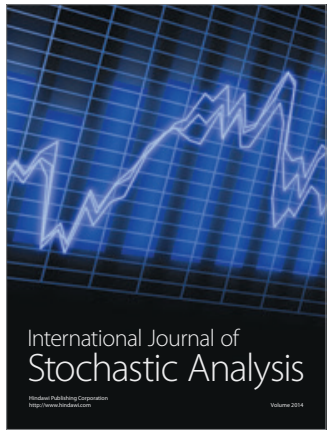

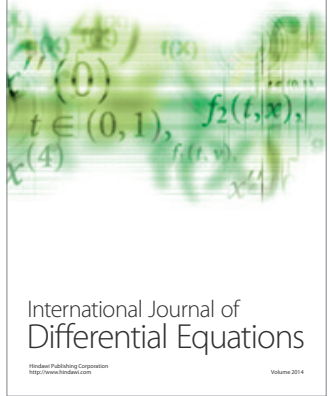
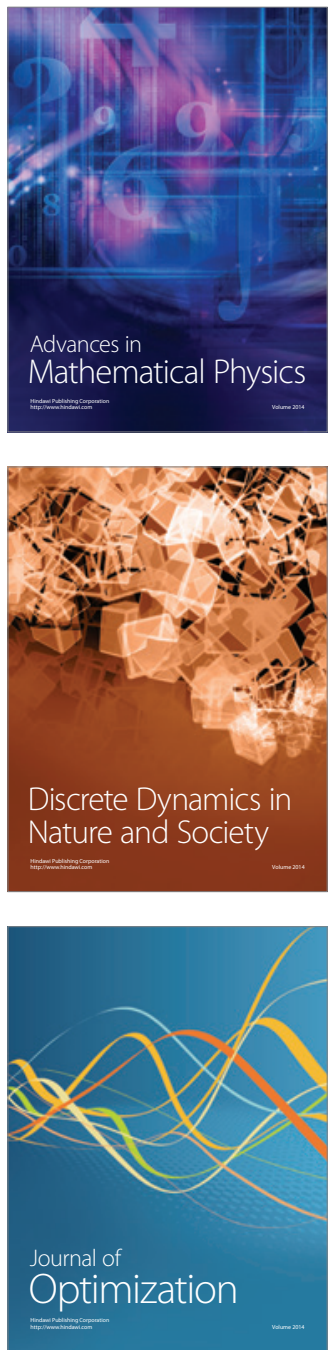